\title{
Adolescents' Attitude towards Masturbation: Practical Study on a Sample of Senior High School Students in the Cape Coast Metropolis of Ghana
}

\author{
Priscilla Commey Mintah, PhD \\ Department of Educational Psychology, Faculty of Educational Studies, \\ University of Cape Coast, Cape Coast, Ghana \\ Kwaku Darko Amponsah, PhD \\ Department of Teacher Education, University of Ghana, Legon, Accra, \\ Ghana \\ Grace Sintim Adasi, PhD \\ Institute of African Studies, University of Ghana, Legon, Accra, Ghana/ \\ Agogo Presbyterian Women's College of Education, Ghana \\ Rosina Okyere Amponsah, MA \& M.Ed. \\ Accra Metro Education Office, P. O. Box 337, Ghana
}

\begin{abstract}
Doi:10.19044/esj.2020.v16n28p195 URL:http://dx.doi.org/10.19044/esj.2020.v16n28p195 Submitted: 13 September $2020 \quad$ Accepted: 13 October $2020 \quad$ Published: 31 October 2020

Copyright 2020 Mintah et al. Distributed under Creative Commons CC-BY 4.0 OPEN ACCESS
\end{abstract}

\begin{abstract}
There are societal variations as to how masturbation is regarded because some societies regard it as a religious or cultural bizarre, while others tolerate it on biological grounds. This study employed a descriptive survey design to explore adolescents' attitudes towards masturbation in selected senior high schools in the Cape Coast Metropolis of Ghana. The target population was formed by form two students in both schools. The total population for the second-year students in the boys' school was 509, while the second-year students in the girls' school were 496. Specifically, it was carried out in two single-sex Senior High Schools (SHS) in the Cape Coast Metropolis. Purposive sampling was employed to select single-sex schools in the metropolis, as they are noted for the practice of masturbation. Three hypotheses on masturbatory practices, attitudes toward masturbation and masturbatory practices and self-concept were formulated for the study. The results indicated that implicitly, both sexes' masturbatory practices are similar. Both sexes also have similar attitude towards masturbation; as well as the masturbatory practices and selfconcept in the Cape Coast Metropolis. Similarly, it was found that the attitude of both sexes towards masturbation was characterised by ambivalence, as they regarded it to be an
\end{abstract}


irreligious and bad practice, yet, they covertly practised it. It was recommended that stakeholders should give adolescents thorough sexual education without expressing their prejudices concerning masturbation. They should be made to understand that sexual urges are a natural part of their development that must, however, be controlled for moral and spiritual reasons.

Keywords: Adolescents, females, senior high school students, males, masturbation.

\section{Introduction}

Attitudes toward masturbation, or genital sexual self-stimulation, differ greatly across cultures. Some cultures accept or even promote masturbation throughout childhood and teenage years, while others, at any age, condemn this activity. Nevertheless, almost all human cultures show some criticism of adult masturbation, from minor mockery to harsh penalties. However, it seems that at least some adults in all cultures do it (Gelbal \& Duyan, 2006). Masturbation is not a topic frequently and overtly discussed either in families or at school. Masturbation risks lead to psychological problems, irreversible physical harm, and temporary disorders such as eye problems or acne that have culminated in the conclusion that masturbation is bad or dirty (Ballan, 2012).

In the majority of the African cultures, masturbation is frowned upon and, therefore most adolescents who engage in it have feelings of guilt of doing something they have been made to believe is bad. Byers, Nichols, Voyer, and Reilly (2012) observed that even though there is no obvious effort to avoid masturbation, the fear of the mother is transmitted to the child utilizing compassion, creating a subsequent sense of unease within him. If the mother's anxiety level is high, the child can detach or remove the actions that triggered the anxiety from awareness. Despite this, some children grow into adolescence and continue to practice it. Byers, Nichols, and Voyer (2013) generally claim that masturbation behaviours are of a varied type. Attitudes in America reflect the effect of two major sources: the history of Judeo-Christianity and, at present, the rejected medical opinion formed more or less in line with this history. Today, nevertheless, many religionists are more likely to forgive and support masturbation while not able to accept it as ethical. These theological and moral values have affected medical choices for many years, and still today (Byers et al., 2013).

Despite the adverse perceptions of masturbation, De Looze, Constantine, Jerman, Vermeulen-Smit, and Ter Bogt (2014) contended that there is some evidence in adolescents that suggest there is a link between the report of masturbation and positive self-esteem. One may speculate that adolescents who have a better understanding of themselves and those who are more comfortable with their self-esteem masturbate (De Looze et al., 
2014). Despite widespread masturbation reports notably among adolescent boys (De Looze et al., 2014; Dekker, Van Der Vegt \& Visser, 2015), cultural and religious prohibitions against this activity have persisted. Such taboos have been retained although many practitioners and investigators claim that masturbation promotes self-exploration, self-image development, and ego borders establishment (De Looze et al., 2014; Dekker, Van Der Vegt \& Visser, 2015). In Dekker's view, Van Der Vegt and Visser (2015) not only view masturbation in a positive light, but some also suggest that its lack of use can be related to psychological challenges.

The cross-cultural confirmation of people's behaviours in other cultures shows that with a few exceptions, adults participate in self-genital stimulation (Byers et al., 2012). For instance, the authors noticed that the experimenting of Cubeo (Indians) teenagers is openly displayed as sexual methodologies for children and youth, and performed masturbation with no embarrassment. This notwithstanding, attitudes towards masturbation in the view of Ballan (2012) are now considerably more positive, and only a few people would now subscribe to the practice as a religious or cultural abnormality. By 1970, only about 15 percent of young people believed that masturbation is wrong. Indeed, they emphasised that masturbation is now recommended as a remedy in sex therapy.

Some theories have complied with the view that masturbation is not to be considered as a bizarre or astound practice. For example, the Psychoanalytic Theory considers sex to be one of the main forces of human life. The theory holds that with puberty, sexual desires reignite, and when sexual desires grow increasingly explicitly genital, the oral, anal, and genital tendencies begin to merge with each other to facilitate the biological role of reproduction, the youngster proceeds into the genital stage. Boys will thus directly stimulate their penis and girls will manually stimulate their clitoris or rub their genital area against things (Fortenberry, 2013). The Cognitive Learning Theory, on the other hand, holds that the adolescent can develop masturbatory practice as a higher-level order and reasoning because he or she may be under the strict supervision of parents and may not get the opportunity to satisfy his or her sexual desires. As a result, a higher-level order to satisfy this desire will be to masturbate. Both sexes could also fear pregnancy and therefore entertain masturbatory practices (Dewinter, Vermeiren, Vanwesenbeeck, Lobbestael \& Van Nieuwenhuizen, 2015).

In general terms, the theory of social learning identifies emulation and identity as two mechanisms that are useful in understanding one's sense of masculinity or femininity. The theory argues that it is common knowledge that adolescents imitate those they see as heroes and heroines. In terms of masturbation, this theory postulates that male and female adolescents express their joy in the practice as they practice it with their pals, usually the fresh 
students. The fresh students, on the other hand, imitate these acts by practicing it with their classmates or other friends. There are cases whereby, adolescents may see their peers masturbate openly and this will necessitate the desire to imitate what their peers are doing (Hellemans, Colson, Verbraeken, Vermeiren \& Deboutte, 2007).

Comparatively, the Sexual Script Theory emphasises on previous sexual acts or experiences concerning when and where the act or experience was perpetrated. So, scripts are strategies people hold in their minds. It takes into account what their current responsibility is and what they will be undertaking in the future. Often, they are instruments that help people recall what they have done previously. Thus, a teenage boy may remember how pleasurable it was the first time he masturbated and this may urge him to continue the practice whenever he is alone (Hellemans, Roeyers, Leplae, Dewaele \& Deboutte, 2010).

\section{Statement of the Problem}

There are societal variations as to how masturbation is regarded. In some societies, masturbation is either regarded as a religious or cultural bizarre, while in other societies it is tolerated on biological grounds. Whatever may be the case, most societies worldwide have a phobic tendency towards the practice. Thus, anyone who indulges in the practice is seen as a cultural misfit, immoral, irreligious, or in spiritual terms is considered as demon-possessed. Nevertheless, some societies have greeted the practice with enormous ambivalence. In such societies, there is a dilemma among perpetrators of the practice, who regard it as sinful, immoral, or culturally unacceptable and on the other hand, they cannot help but carry out the act because they do not have sexual partners, yet their biological needs must be satisfied. This raises questions as to whether it is appropriate to widely and publicly condemn masturbation, while perpetrators carry out the practice underground. How can the situation, therefore, be harmonised, whereby the practice is overtly condemned and not practiced covertly?

Some parents are so programmed in the Ghanaian sense that the word masturbation stirs up indignation and apprehension. Unavoidably, when they find a baby touching its genitals, the mother or other significant individuals respond with fear, panic, or frustration. The hands of the baby are smacked or detached from its genitalia. Thus criticism is verbally or nonverbally conveyed. Persistence of such trends over a period of time can lead the child to exclude genital feelings from his consciousness, rendering a significant characteristic of himself denied. Holistically, this may culminate in the infant developing a phobic attitude towards sex or sexual activities when he or she transits the adolescence and eventually grows up to become an adult (Holmes \& Himle, 2014). This phobic attitude espouses the Sociological 
Theory of Sexuality which opines that cultures define what is right and wrong sexually. Therefore, people in cultures that embrace masturbation invariably have a positive attitude towards the practice, whereas, in cultures that abhor masturbation, there is the tendency for people to have a negative attitude towards the practice.

The arguments reveal that in one way or the other, most societies either display a religious or cultural phobic towards masturbation. This is a matter of concern as to whether it is the same phobia that is equally displayed among adolescents who are even more susceptible to the practice because of their budding sexual urges or drives during adolescence with the tendency to exercise very little or no self-control. This raises further concerns as to whether or not the thought and practice of masturbation have been completely deleted from the minds of adolescents. If indeed they regard it as a phobia or they rather covertly practice it because as Fortenberry (2013) argued one of the significant powers in human life is sexuality. This interrogation is the basis of this article. Arguments on adolescence and masturbation shall be broadened by a theoretical framework. Following this framework, the methodological components, empirical evidence, conclusions, and policy implications of the study shall be addressed.

\section{Theoretical Framework on Adolescence and Masturbation}

Several researchers like Sanders (2004); Gelbal and Duyan (2006); Ballan (2012); Byers et al. (2012); Liddon, Michael, Dittus, and Markowitz (2013); Byers et al. (2013); De Looze et al. (2014) have theorised on adolescence and masturbation which in their view is considered a cultural or religious bizarre but is however feasible, as sex is regarded as a natural phenomenon of life. This notwithstanding, masturbation is seen as more exploratory and therefore more intense during adolescence because, at this stage, there is a surge in sexual drives and urges in every human being.

The term adolescence denotes specifically to a communally defined duration in which individuals adapt to the physical, psychological, and societal variations connected with the changeover from childhood to adulthood (Sanders, 2004). Adolescence is a developmental change from a child's actions and attitudes to an adult's habits, attitudes, and obligations. It is also the period of one's life during which one develops from being a child into an adult (Liddon et al., 2013). Adolescence, a transitional period between childhood and adulthood, has seen various choices in addressing its definition. In this case, adolescence starts with the beginning of puberty (with sexual maturity and readiness to recreate) and concludes with the completion of physical development, which can be described in biological terms usually late in the teen years (Tolman, \& Diamond, 2014). Others, who perceive adolescence psychologically, regard it as the development of 
cognition, feelings, and behaviour that characterise the stage. Be it psychological or biological an adolescent may be seen as a young person between the ages of twelve and twenty (Holmes \& Himle, 2014).

Adolescents the world over have biological and sexual needs like hunger, activity, thirst, sex among others. Sex needs appear to be the most crucial and challenging of all the needs they encounter. In describing adolescents' sexual behaviour, Nichols and Blakeley-Smith (2009) emphasized that the kinds of sexuality that are awakened in the adolescent results in increased nervous excitement, anxiety, and other personality disturbances. The combination of numerous physical changes during puberty, such as sexual desire, adult physiology, and changes in social roles, such as anticipated adult behaviour and one's place in peer groups, significantly accounts for adolescent sexual anxiety experimentation. During puberty, the sexual thoughts, desires, and actions that are present in life are frequently amplified. Mollborn and Everett (2010) explained that adolescence begins with puberty, the time when one is maturing sexually. Puberty offers obvious, convincing proof of physical development, apparent masculinity or femininity, and reproducibility.

Going by Mehzabin and Stokes (2011), most teenagers who pass through puberty are keenly conscious of physical changes and are anxious with any variations they view as odd. They appear to overdo and obsess about physical symptoms, though they may have trouble expressing their worries. Adolescents have rapid, large mood swings, become easily frustrated and angry and switch between intense collaboration and severe opposition to guidance from adults. Mehzabin and Stokes (2011) subsequently emphasized that adolescents form close relationships with colleagues socially, and may encounter some issues out of fascination. Sexually, with menarche activities, teenagers will rediscover masturbation, a satisfying self-stimulation of their sexual organs to achieve satisfaction, and maybe practicing it with varying frequency especially when they learn about varieties of sexual expressions.

Masturbation is a practice among some adolescent males in boarding schools to take pillows as their lovers and in their deep fantasies rub their penis on the pillow to achieve orgasm. Some openly joke about masturbation using jargon to refer to masturbation. In other cases, some boys may masturbate in groups in bathrooms whereas some girls also fantasise scenes from pornographic films and masturbate secretly (Gelbal \& Duyan, 2006). Udry (1988) claimed that two-thirds of young girls and nearly all boys would masturbate into orgasm by the end of adolescence. Ahmad (2004) resonated with the fact that there is hardly any young man who does not masturbate at one time or the other. These studies make it significant to comprehend the 
function of masturbation in the growth process to comprehend normal development since masturbation may begin in infancy.

Ballan (2012) suggests that masturbation begins in childhood. The child, as the first step towards mastery of themselves and of their environment, starts to discover the world around them. Normally, in terms of their eyes, arms, legs, and, finally, genitals, their body offers a ready source of manipulable items. Early selection of the genitals for exploration is random, but the pleasurable sensation is for the infant, a new way of experiencing his or her body. His or her interest, however, is transient as other aspects of the world claim his or her attention. Therefore, the probability that masturbation increases with an adolescent age or during adolescence is somewhat debatable as sexual practices during this stage are less narcissistic and are more geared at and fascinated towards the opposite sex. This is the position of the Psychoanalytical Theory (Fortenberry, 2013).

The Psychoanalytical Theory takes the view that sexuality becomes less self-oriented in the genital stage than it was in infancy, and as suitable sexual imagery is oriented towards other individuals. The theory augments that sex as one of human life's key forces (Fortenberry, 2013), defined human personality as being divided into three main parts; id, ego, and superego. The id is the essential aspect of personality and is congenital. It is the source of psychic energy, it includes the impulses, as it only functions on the concept of gratification and consequently can be very unreasonable. The ego, on the contrary, works on the basis of truth and attempts to hold the id in line. The superego's purpose is for the consciousness to suppress the id's urges and convince the ego to aspire for ethical objectives rather than practical goals. De Looze et al. (2014) found that sexual desires rejuvenate with puberty and that the infant is heading into the genital stage. During this stage, sexual impulses become much more explicitly, genital, and the oral, anal, and genital desires begin to merge together to facilitate reproductive biological function. Despite these challenges, genital self-stimulation and arousal also happen in boys and girls during the genital period, although they are comparatively less evident throughout the life span than other periods. Males will actively arouse their penis, and girls will indirectly arouse their clitoris or rub their genital area against kinds of stuff.

Dewinter et al. (2015) noted that, during adolescence, a wave of sexual desire occurs through puberty. This increased sexuality can be triggered by several factors such as body changes, self-awareness, increased levels of sex hormones, increased cultural focus on sex and the practice of gender roles for adults, as well as an enhanced ability to think in an abstract manner, which Dewinter et al. (2015) termed as cognitive learning. The writers theorised on this method of learning, arguing that as an adolescent, he or she learns the ability to think independently, identify and merge things in 
a more complex way, entering the formal operational stage, which is an open-ended period, starting at the age of eleven. It is during this point that the infant grows higher-level order and critical thinking skills.

The adolescent can develop masturbatory practices as a higher-level order and reasoning because it is the stage in life where one's sexual drive is high and needs more self-control to overcome it. The adolescent may also happen to be under the strict supervision of parents and may not get the opportunity to satisfy his or her sexual desires. Thus, a higher-level order to satisfy this desire will be to masturbate. On the other hand, where there is the freedom to enjoy sex, the fear to be pregnant on the part of girls or get a girl pregnant on the part of boys also calls for masturbation (Dekker et al., 2015).

Associated with high-level order and reasoning is the desire for imitation and recognition, two mechanisms that are useful to understand the formation of gender identity and one's understanding of masculinity or femininity. Thus, considering masturbation, male and female adolescents express their joy in it as they practice it with their pals usually the fresh students through imitation. Those who masturbate, getting to know of others who do the same, form groups, and operate together. This helps them to share ideas about various techniques that can be used to satisfy oneself or the other especially for the females (Hellemans et al., 2007).

Aside from imitating seen events, every human being tends to recall past events. Hellemans et al. (2010) termed this as Sexual Scripts in which human sexual behaviour is not spontaneous. Rather, humans learn an intricate script that tells us who, what, where, and why we are sexually doing what we are doing. For instance, what part of the script tells us that sex should occur with someone else, around one's age, and on his own? The script would ask the question when one should, for example, masturbate, at night, afternoon among others. 'The where script', may talk about one masturbating in the bathroom or bedroom. It even scripts masturbation. Scripts are therefore strategies that people hold in their heads. It takes into account what they are doing, and what they will be doing. They are also tools that assist individuals recall what they did previously (Hellemans et al., 2010). Thus, a teenage boy may remember how pleasurable it was the first time he masturbated and this may urge him to continue the practice whenever he is alone.

Comparatively, De Graaf, Kruijer, Van Acker and Meijer (2012) postulated that culture plays a role in people's attitudes towards masturbation. In line with this argument, the writer holds in the Sociological Theory of Sexuality that sexuality is strongly related to cultural values. So communities carefully determine what usual and unusual sexual activities are, and which are right and wrong. Some cultures define masturbation as abnormal, whereas others define it as normal, but the point is that all cultures 
define it one way or the other. Similarly, Attwood, Hénault, and Dubin (2014) claim that some cultures take a permissive attitude toward masturbation for males and females, and some are permissive for neither. Going by the writer, a culture's ideologies describe what is sexually immoral and what is right. Therefore, in cultures whereby masturbation is abhorred, there is a tendency for people to have a negative attitude towards it, whereas cultures that embrace it would experience a positive attitude towards it.

Relatedly, in aligning the cultural perspectives of masturbation to behaviour, Moore and Rosenthal (2006) posited in the Problem Behaviour Theory (PBT) that problem behaviours are behaviours which deviate from the cultural and legal rules of society and trigger social control and external sources. Traditional habits are those which are anticipated and accepted socially and in a regulative manner. The theory opined that the concept of personality requires a combination of constant, lasting emotional impacts such as the function of motivation-instigation, defined by the importance put on accomplishment and freedom. Contrarily, the nature of personal values is related to an individual's sense of self, compared to society and personal control system, which gives an individual reason not to engage in problem behaviour. Moore and Rosenthal (2006) found that problem behaviour is often linked to increased social acceptance in a perceived environment; peer models; low parental power, help, and impact; parental and peer standards are incompatible.

The PBT suggests that behavioural issues become apparent when the personality system and the perceived environment system collide. The central features of the adolescent personality are impulsiveness, risk-taking, assumed invincibility, striving to find social identity, inconsistencies in thought due to being trapped into conventional peer culture; resistance against authority combined with disruptions in psychosocial adjustment, inconsistent with cultural and social standards and expectations (such as abstinence from intoxication).

Still, in line with behaviour, the Theory of Planned Behaviour (TPB) opines that Behaviour is based on and steered by three types of cognitive beliefs and consequences, namely: behavioural, normative, and control beliefs. The behavioural beliefs are those concerning the anticipated or likely results of the behaviour that yields a favourable or unfavourable behavioural attitude (outcome). Contrarily, they are normative beliefs regarding what others anticipate (normative expectations) as well as the person's willingness to pursue those aspirations. These convictions result in a level of societal force to meet social standards. The teenager believes others like peers want them to perform the behaviour. However, beliefs regarding the ease or difficulty of conducting behaviour, which results in the level of apparent behavioural control, are beliefs of control (Byers et al., 2012). Additionally, 
the writers opined that the attitude towards the behaviour of each of these beliefs and results are taken into consideration. Consequently, the subjective norm and apparent behavioural control come together to decide the intent of the behaviour. For instance, the more beneficial the behavioural attitude, the more beneficial the subjective norm, and the higher the recognisable behavioural control, the greater the behavioural performance prospects of the specific intent.

Byers et al. (2013) advance in the Social Norms Theory (SNT) that subjective norms or purported aspirations of others or peer groups that approve or reject a specific action are indicators of that behaviour. The theory contends that individuals are highly impacted by what they think their peers are doing or thinking, and then act in accordance with the norm or social expectation they believe. People can therefore overrate the value of behavioural problems and underrate healthy habits, which contribute to higher problem behaviours. In addition, subjective norms resulting from an inaccurate evaluation of what others are doing will affect social behaviour (Holmes \& Himle, 2014).

\section{Methodology}

The study employed a descriptive survey design to explore adolescents' attitudes towards masturbation in selected high schools in the Cape Coast Metropolis of Ghana. Specifically, it was carried out in two single-sex Senior High Schools (SHS) in the Cape Coast Metropolis. The target population was formed by form two students in both schools. The total population for the second-year students in the boys' school was 509, while the second-year students in the girls' school were 496.

Purposive sampling was employed to purposively select single-sex schools in the metropolis, as they are noted for the practice of masturbation. The hat method of simple random sampling was used to establish a list of single-sex schools in the metropolis. The list was cut out and placed in separate boxes for males and females. A school was randomly selected from each group. St Augustine SHS was selected to represent the male institutions, while Holy Child SHS was chosen to represent female institutions. Robson (2002) recommended 100 observations for major subgroupings in survey research, with twenty to fifty for minor sub-groupings". On this note, 20 students out of every 100 were randomly selected. This generated 80 students per school and gave a total sample of 160 students who were earmarked for the study.

A questionnaire was administered to the students. The Statistical Product and Service Solutions (SPSS) Version 17 was employed to screen, code, and analyse the data. The questionnaire had four sections. The first section described the demographic information of the participants. 
Statements on male and female masturbatory practices were put in section two. In section three, statements on adolescent male and female attitude towards masturbation were made. Section four was made up of statements on adolescent males and females' self-concept and masturbatory practices.

In analyzing the data, the Mann-Whitney test was used to ascertain whether or not there were significant differences in the masturbatory practices of males and females as the data on this section was not normally distributed. Additionally, the parametric conditions of validity were not met in this section. The Independent samples t-test was also used to investigate whether or not there were significant differences between males and females in their attitudes towards masturbation. Finally, the t-test was employed to examine whether or not there were significant differences in the masturbatory practices and self-concept of females and males.

\section{Results and Discussions}

Masturbatory practices among male and female adolescents

This section constitutes the results and discussions from the collected primary data. It examines adolescents' masturbatory practices, their attitude towards masturbation, as well as their masturbatory practices alongside their self-concept. The Mann-Whitney test was adopted to test if there are significant differences in the masturbatory practices of males and females. Given these, it was hypothesised that:

\section{$\mathrm{H}_{01}$ : There is no statistically significant difference between male and female adolescents regarding masturbatory practices.}

Findings from the analyses in Table 1 show that there were no statistically significant differences $(z=-0.048, p=0.0961)$ between the male and female adolescents as far as their masturbatory practices were concerned. The mean ranking for adolescent males was 48.41 , while females reckoned 48.71 as indicated in Table 1. Implicitly, both sexes' masturbatory practices are similar. Thus, the null hypothesis was rejected.

Table 1: Mann-Whitney Test on Adolescent Male and Female Masturbatory Practices

\begin{tabular}{|c|c|c|c|c|}
\hline $\begin{array}{l}\text { Sex } \\
\text { Students }\end{array}$ & of Freq. & $\begin{array}{l}\text { Mean } \\
\text { Ranking }\end{array}$ & Z & P-value \\
\hline Male & 67 & 48.41 & -0.048 & 0.096 \\
\hline Female & 29 & 48.71 & & \\
\hline
\end{tabular}

Source: Field data, 2019 
Researchers have not documented literature on masturbatory practices. However, holistically they explain how boys and girls masturbate by virtue of their sex. Fortenberry (2013), for example, demonstrates that males will directly arouse their penis, and females will manually arouse their clitoris, or use objects to rub their genital region. Moreover, for the sake of adventure, exploration, and excitement, Hellemans et al. (2007) maintained that adolescents masturbate to express their joy in it as they practice it with their pals usually the fresh students. On the other hand, the fresh students imitate these acts by practicing it with their classmates or other friends. There are cases whereby adolescents may see their peers masturbate openly and this will necessitate the desire to imitate what their peers are doing. Those who masturbate, getting to know of others who do the same, form groups, and operate together. This helps them to share ideas about various techniques that can be used to satisfy oneself or the other especially for the females (Hellemans et al., 2007).

Table 2 shows the total number of respondents who admitted to having ever masturbated in support of the hypothesis. Even though there were eighty statements in the questionnaires each for both males and females, 67 (83.7\%) males and 29 (16.3\%) females admitted categorically that they masturbate.

Table 2: Adolescent Males and Females Who Had Masturbated

\begin{tabular}{llclc}
\hline Response & Males Freq. & $\%$ & Females Freq. & $\%$ \\
\hline Yes & 67 & 83.7 & 29 & 16.3 \\
No & 13 & 36.3 & 51 & 63.7 \\
\hline Total & 80 & 100.0 & 80 & 100.0 \\
\hline
\end{tabular}

Source: Field data, 2019

On further inquiry, the respondents revealed the number of times they masturbate per week, the results depicted that more males (67) than females (29) admitted they masturbate. The data shows that 27 (40.3\%) adolescent males and $15(51.8 \%)$ adolescent females masturbate more than six times in a week.

The lower percentage for females who do not masturbate could be attributed to low sexual esteem. This explains the females' willingness in the study to answer questions on general knowledge on masturbation and adolescent source of information on masturbation but left portions on adolescent masturbatory practices, which sought to find out at what age they started masturbating and how many times they masturbated in a week.

Inherent in many of the reasons that accounted for the lower female report on masturbation in this study, are the notion of one's perception about 
masturbation and the fear of being known to have masturbated or to masturbate. This was evident in the manner by which female adolescents in this study carried themselves in filling the questionnaire. Even though confidentiality, anonymity, and non-traceability of respondents' responses were guaranteed, the sensitive nature of the questionnaire led to some female respondents feeling jittery and somewhat reluctant to disclose, private matters.

The females' reluctance to divulge information on when and how often they masturbate could be explained by societal norms that have regarded any sex-related activity to be men's affairs. By so doing, women who willfully engage in any sexual activity are seen to possess a perverse mind. In the PBT, Moore, and Rosenthal (2006) contended that social and legal societal norms play a significant role in the behaviour. The writer articulates that problem behaviour is behaviour that moves away from the standards (social and legal) and triggers exogenous sources of social control and reactions. Similarly, traditional habits are those which are predicted and accepted socially and normatively. Moore and Rosenthal (2006) also noted that problem behaviour in a perceived environment is often related to higher social acceptance, peer pressure, low parental control, assistance, and impact. The incongruity of parental and peer expectations, as may have been the case with masturbating female adolescents in the study, as female sexual agility in the Ghanaian context is considered to be.

\section{Male and female adolescents' attitude towards masturbation}

Furthermore, the Independent samples t-test was used in ascertaining whether or not there existed a statistically significant difference in attitude towards masturbation between male and female adolescents. It was therefore hypothesized with an alpha level of 0.05 that:

\section{$\mathrm{H}_{02}$ : There is no statistically significant difference between male and female adolescents' attitudes towards masturbation.}

Table 3 presents the relevant figures to support the prediction. The mean score for males was 1.99 and females 1.87. These figures do not differ statistically, signifying that there was no significant difference existing between adolescent males and females' attitudes towards masturbation. The results in Table 3 show that there was no significant difference $(t=.900$, $\mathrm{p}=.370$ ) between both sexes' attitude towards masturbation. This warranted the rejection of the null hypothesis. This notwithstanding results from the analysis portray that whilst a substantially high percentage of males $(85.0 \%)$ and females (78.8\%) strongly believed that masturbation is sinful, quite close percentages of $86.3 \%$ for males and $83.8 \%$ for females saw masturbation as a bad practice. 
Table 3: Independent Samples T-Test Comparison of Adolescent Male and Female Attitude towards Masturbation

\begin{tabular}{lccccc}
\hline $\begin{array}{l}\text { Sex of } \\
\text { students }\end{array}$ & Freq. & Mean & SD & T & P \\
\hline Male & 67 & 1.99 & .594 & .900 & .370 \\
Female & 29 & 1.87 & .758 & & \\
\hline
\end{tabular}

Source: Field data, 2019

Thus, this contradicts the revelations that 67 (83.7\%) males and 29 (16.3\%) females admitted categorically that they masturbate. Emphatically, this portrays that while the approval of masturbation is now explicit; some adolescents still have mixed feelings about it.

Peer pressure could be a supporting factor for the adolescents' ambivalence of masturbation. This corroborates Byers et al. (2012) position in the TPB that normative expectations are beliefs in what others anticipate and the individual's yearning to live up to those anticipations are normative beliefs. These convictions result in a level of social pressure to meet the norms. The teenager believes others like peers want them to execute the behaviour. Contrarily, beliefs about the ease or difficulty of conducting behaviour, which results in the levels of social behavioral control, are beliefs of control (Byers et al., 2012). The writer adds that the subjective norm and perceived control of behavior combine to determine the intent of behaviour. For instance, the more peer pressure mounted on an adolescent to masturbate, the more he or she does and desires the practice and regards it as normal and practices it with ease without any feeling of guilt.

At a significant level of 0.05 , the Independence Samples t-test was again employed to test whether or not there were significant differences in the masturbatory practices and self-concept of adolescent males and females. Thus, the null hypothesis was formulated predicting that:

\section{$\mathrm{H}_{03}$ : There is no statistically significant difference between adolescent males and females regarding their masturbatory practices and self- concept.}

The results are presented in Table 4 . The results of Table 4 reveal that there was no statistically significant difference $(\mathrm{t}=1.258, \mathrm{p}=.211)$ between adolescent males and females' masturbatory practices and their self-concept. 
Table 4: Comparison of Adolescent Males and Females' Self-concept and Masturbatory Practices

\begin{tabular}{|c|c|c|c|c|c|c|}
\hline $\begin{array}{l}\text { Sex } \\
\text { students }\end{array}$ & of & Freq. & Mean & SD & $\mathrm{T}$ & $\mathrm{p}$ \\
\hline Male & & 67 & 1.89 & .472 & 1.258 & .211 \\
\hline Female & & 29 & 1.79 & .468 & & \\
\hline
\end{tabular}

Source: Field data, 2019.

This is because the mean scores for males were 1.89, while the females registered 1.79, which did not differ statistically. By implication, the masturbatory practices and self-concept of both sexes were similar. Thus, the null hypothesis was also rejected. This suggests that males and females have the similar masturbatory practices and self-concept.

\section{Conclusion and Recommendations \\ Conclusion}

The evidence suggests that there were no significant differences in the masturbatory practices; attitude towards masturbation; as well as the masturbatory practices and self-concept of male and female adolescents in the Cape Coast Metropolis. This suggests that there are similarities between both sexes in their masturbatory practices, self-concept, and attitude towards masturbation. Additionally, the results and discussions suggest that the attitude of both sexes towards masturbation was characterised by ambivalence, as they regarded it to be an irreligious and bad practice, yet, they covertly practised it. However, their ambivalence may be by a surge in their sexual desires and urges at the adolescence stage of development; limited parental control and more peer pressure; the fear of pregnancy, yet their desire to gratify their sexual yearnings.

\section{Recommendations}

As a measure to pre-empt the covert practice of masturbation, the adolescents should be given thorough sexual education by their parents, religious and educational institutions, the media, and other policy-making institutions (governmental and non-governmental) whose activities hover around sexual education without any form of intimidation or creating a phobic or awful feeling about the practice. Therefore, masturbation should be discussed overtly by these stakeholders without attaching any biases to the practice. Adolescents should be made to understand that sexual desires are a natural part of their development, which must, however, be controlled to ensure moral decadence and spiritual purity. They should also be advised by stakeholders to focus on their career and educational goals, visions and 
building their life skills (including enhancing their natural talents and gifts and spiritual life) during their adolescence stage of development. This will enable them to think less about sexual activities and concentrate on building a promising and better secular and spiritual future for themselves.

\section{References:}

1. Ahmad, S. D. (2004). Masturbation. Retrieved on December 9, 2019, from http://www. homeoint.org/site/ahmad/masturbation.htm.

2. Attwood, T., Hénault, I., \& Dubin, N. (2014). The autism spectrum, sexuality, and the law: What every parent and professional needs to know. London: Jessica Kingsley Publishers.

3. Ballan, M. S. (2012). Parental perspectives of communication about sexuality in families of children with autism spectrum disorders. Journal of autism and developmental disorders, 42, 676-684.

4. Byers, E. S., Nichols, S., Voyer, S. D., \& Reilly, G. (2012). Sexual well- being of a community sample of high-functioning adults on the autism spectrum who have been in a romantic relationship. Autism, 17(4), 418-433.

5. Byers, E. S., Nichols, S., \& Voyer, S. D. (2013). Challenging stereotypes: Sexual functioning of single adults with high functioning autism spectrum disorder. Journal of Autism and Developmental Disorders, 43, 2617-2627.

6. De Graaf, H., Kruijer, H., Van Acker, J., \& Meijer S. (2012). Sexual health among youths in the Netherlands. Delft: Eburon.

7. Dekker, L. P., Van Der Vegt, E. J. M., \& Visser, K. (2015). Improving psychosexual knowledge in adolescents with autism spectrum disorder: pilot of the tackling teenage training program. Journal of Autism and Developmental Disorders, 45(6), 1532-1540

8. De Looze, M., Constantine, N., Jerman, P., Vermeulen-Smit, E., \& Ter Bogt, T. (2014). Parent adolescent sexual communication and its association with adolescent sexual behaviours: A nationally representative analysis in the Netherlands. Journal of Sex Research, 52(3), 257-268.

9. Dewinter, J., Vermeiren, R., Vanwesenbeeck, I., Lobbestael, J., \& Van Nieuwenhuizen, C. H. (2015). Sexuality in adolescent boys with autism spectrum disorder: self-reported behaviours and attitudes. Journal of Autism and Developmental Disorders, 45(3), 731-741.

10. Fortenberry, J. D. (2013). Sexual development in adolescents. In Bromberg, D. S., O'Donohue, W.T., (Eds). Handbook of Child and Adolescent Sexuality: Developmental and Forensic Psychology. Oxford: Elsevier Ltd. p. 589. 
11. Gelbal, S., and Duyan, V. (2006). Attitudes of university students toward lesbians and gay men in Turkey. Sex Roles, 55, 573-579.

12. Hellemans, H., Colson, K., Verbraeken, C., Vermeiren, R., \& Deboutte, D. (2007). Sexual behaviour in high-functioning male adolescents and young adults with autism spectrum disorder. Journal of Autism and Developmental Disorders, 37(2), 260-269.

13. Hellemans, H., Roeyers, H., Leplae, W., Dewaele, T., \& Deboutte, D. (2010). Sexual behaviour in male adolescents and young adults with autism spectrum disorder and borderline/mild mental retardation. Sexuality and Disability, 28(2), 93-104

14. Holmes, L. G. \& Himle, M. B. (2014). Brief report: Parent-child sexuality communication and autism spectrum disorders. Journal of Autism and Developmental Disorders, 44(11), 2964-2970

15. Liddon, N., Michael, S. L., Dittus, P., \& Markowitz, L. E. (2013). Maternal underestimation of child's sexual experience: Suggested implications for HPV vaccine uptake at recommended ages. Journal of Adolescent Health, 53(5), 674-676

16. Mehzabin, P. \& Stokes, M. (2011). Self-Assessed sexuality in young adults with high functioning autism. Research in Autism Spectrum Disorders, 5(1), 614-621.

17. Mollborn, S. \& Everett, B. (2010). Correlates and consequences of parent- teen incongruence in reports of teens' sexual experience. Journal of Sex Research, 47(4), 314-329.

18. Moore, S. \& Rosenthal, D. (2006). Sexuality in adolescence: Current trends. London and New York: Routledge.

19. Nichols, S. \& Blakeley-Smith, A. (2009). Working with families towards facilitating healthy sexuality for individuals with autism spectrum disorders. Social Work in Mental Health, 8(1), 72-91.

20. Robbins, C. L., Schick, V., Reece, M., Herbenick, D., Sanders, S., Dodge, B., \& Fortenberry, J. D. (2011). Prevalence, frequency, and associations of masturbation with partnered sexual behaviours among US adolescents. Archives of Pediatrics and Adolescent Medicine, 165(12), 1087-1093.

21. Robson, C. (2002). Real world research: A resource for social scientists and practitioner-researchers, $\left(2^{\text {nd }}\right.$ ed.) United Kingdom: Blackwell Publishing Ltd.

22. Sanders, S. A. (2004). Human sexuality. Microsoft Encarta, WA: Microsoft Corporation. 
23. Tolman, D. L., \& Diamond, L. M. (2014). APA handbook of sexuality and psychology. Washington, DC: American Psychological Association.

24. Udry, J. (1988). Biological predisposition and social control in adolescent sexual behaviour. American Sociological Review, 53, 709722 